\title{
CONOCIMIENTOS, ACTITUDES Y PRÁCTICAS SOBRE LAS GEOHELMINTIASIS EN UNA COMUNIDAD RURAL DE COLOMBIA
}

\author{
María del Pilar Díaz Murillo, Antropóloga. M.Sc. ${ }^{1}$, Ligia Inés Moncada Álvarez \\ Bióloga M.Sc. ${ }^{2}$, Patricia Reyes Harker Md. M.Sc. ${ }^{3}$, Julián Alfredo Fernández Niño Md. ${ }^{4}$, \\ Diego Fernando Cano Rosales, Md. ${ }^{4}$ Y Rosa Suárez Prieto, Psicóloga ${ }^{5}$. \\ ${ }^{1}$ Msc. Salud Pública, Profesora Asociada, Departamento de Salud Pública, Facultad de Medicina, Universidad \\ Nacional de Colombia. Bogotá, Colombia. ${ }^{2}$ M.Sc. Microbiología, Profesora Titular, Departamento de Salud \\ Pública, Facultad de Medicina, Universidad Nacional de Colombia. Bogotá, Colombia. ${ }^{3}$ M.Sc. Medicina \\ Tropical, Profesora Asociada, Departamento de Salud Pública, Facultad de Medicina, Universidad Nacional \\ de Colombia. Bogotá, Colombia. ${ }^{4}$ Universidad Nacional de Colombia. ${ }^{5}$ Profesora Asistente, Departamento de \\ Salud Pública, Facultad de Medicina, Universidad Nacional de Colombia. Bogotá, Colombia.
}

\section{Resumen}

Las geohelmintiasis son un grupo de enfermedades desatendidas que afectan especialmente a la población infantil de países en vía de desarrollo. En este artículo se muestran los resultados de un trabajo cuyo objetivo fue explorar y describir los conocimientos, actitudes y prácticas sobre las geohelmintiasis en niños y padres de una población rural colombiana, como base para el diseño de un programa de educación en salud. Este es un estudio cualitativo, etnográfico, realizado en la población de La Virgen, (Colombia), cuya validez estuvo dada por la triangulación y su confiabilidad por la concordancia interpretativa, mediante la participación de varios investigadores. Se utilizaron las técnicas de encuesta, entrevistas, grupos de discusión, círculos de reflexividad y observación participante. Los datos de la encuesta se procesaron con el Programa EpiInfo VI y los cualitativos con el Atlas-ti 5.0.66. Aunque los participantes identificaron los factores de riesgo para las geohelmintiasis, no asociaron la infección con contaminación del suelo con los huevos o larvas de los helmintos, pero si con otras acciones, como el consumo de guayaba. Identificaron como manifestaciones clínicas el bruxismo, dolor abdominal, diarrea, y una entidad popularmente denominada "rebote de lombrices". Ellos emplean terapéuticas tradicionales como el paico (Chenopodium ambrosioides), pero también acostumbran a llevar los niños al médico cada seis meses para la desparasitación. Este estudio muestra que los factores de riesgo no deben ser exclusivamente considerados en términos de individuos o de determinantes sociales desarticulados (sexo, edad, nivel socioeconómico), sino dentro de un contexto sociocultural con un potencial explicativo fundamental que permite entender las condiciones bioculturales en que se instalan y prosperan estas infecciones.

Palabras clave: conocimientos, actitudes, prácticas en salud, helmintiasis, escolares.

\section{KNOWLEDGE, ATTITUDES AND PRACTICES ABOUT THE SOIL TRANSMITTED HELMINTHS IN A RURAL COMMUNITY OF COLOMBIA}

\begin{abstract}
Geohelminthiasis are a group of soil-transmitted parasitic diseases that specially affect children in the developing world. This article shows the results of a work which aimed to explore and to describe the

* Correspondencia: Patricia Reyes Harker preyesh@unal.edu.co. Dirección postal: Carrera 30 No. 45-03. Ciudad universitaria. Edificio Facultad de Medicina No. 471. Tercer piso. Oficina 318. Universidad Nacional de Colombia, Bogotá D.C., Colombia. Teléfono: 3165000 Ext: 15032/33 o 15073. Telefax: 3165000. Ext. 15026.

Recibido: Abril 28 de 2010 Aceptado: Junio 20 de 2010
\end{abstract}


knowledge, attitudes and practices regarding geohelminthiasis in children and parents from a Colombian rural area, in order to design a health education program. It was an ethnographic, qualitative study, undertaken in La Virgen, Cundinamarca, Colombia. The validity was given by the triangulation and its confiability from the interpretative concordance using the information obtained from different sources and researchers. The techniques used were interviews, survey, discussion groups, reflexivity groups and observations in situ. Survey data were processed in EpiInfo VI and interviews data were organized using Atlas-ti 5.0.66. Participants identified the risk factors for geohelminth infection, but they do not associate soil contamination with helminths eggs or larvae with the risk to acquire the infection, instead of that they think the infection could be acquired by eating guava, because they think guava worm is alike a geohelminth. Participans identified as clinical signs bruxism, abdominal pain, diahrrea, and an illness called "rebote de lombrices" (sort of "worm riot") by people. Mothers use some traditional therapeutics like "paico" (Chenopodium ambrosioides), but they also use to visit the physician at least twice a year, to get medicine against geohelminths. This study shows that risk factors should not be considered solely in terms of individuals or disjointed of social determinants (sex, age, socioeconomic status), but within a societal context with a fundamental explanatory potential that allows understanding bio-cultural conditions where are installed and thrive these infections.

Keywords: knowledge, attitudes, practices, helminthiasis, school children.

\title{
CONHECIMENTOS, ATITUDES E PRÁTICAS DE MORADORES DE UMA COMUNIDADE RURAL DA COLÔMBIA SOBRE AS GEOHELMINTOSES
}

\begin{abstract}
Resumo
As geohelmintoses pertencem ao grupo das doenças negligenciadas, que afetam especialmente a população infantil nos países em desenvolvimento. Neste trabalho, mostra os resultados de um estudo que visa explorar e descrever os conhecimentos, atitudes e práticas sob as geohelmintoses na Colombia em uma comunidade rural nos pais e crianças como base para projetar um programa de educação em saúde. Esta é uma pesquisa qualitativa, etnográfica, realizada no povoado de La Virgen, (Colômbia), cuja validade foi dada pela triangulação ea sua fiabilidade pela coerência de interpretação, com a participação de vários pesquisadores. Nós usamos técnicas de inquérito, entrevistas, grupos focais, os círculos de reflexão e observação participante. Os dados do inquérito foram processados com o programa Epilnfo VI e os dados qualitativos com o 5,0 Atlas-ti. Os participantes identificaram os fatores de risco para geohelmintoses, embora não associando com a contaminação do solo com ovos ou larvas de helmintos, com o risco de infecção, porem associando com outras ações, tais como o consumo de goiaba. Foram identificadas manifestações clínicas como "bruxismo", dor abdominal, diarréia, e apareceu uma doença conhecida popularmente como "rebote de lombrices". Eles usam as terapias tradicionais, como paico (Chenopodium ambrosioides), mas também tendem a levar as crianças ao médico a cada seis meses para a desparasitação.Este estudo mostra que fatores de risco não devem ser apenas considerada em termos de indivíduos ou desarticulados determinantes sociais (sexo, idade, status socioeconômico), além disso dentro de um contexto cultural com potencial explicativo fundamental que nos ajuda a compreender as condições culturais em que essas infecções se estabelecem e se desenvolvem.
\end{abstract}

Palavras-chave: conhecimentos, atitudes, prática em saúde, helmintoses, educação infantil 


\section{Introducción}

Las helmintiasis transmitidas por el suelo continúan siendo un problema de salud pública relevante en los países en vía de desarrollo (1). Se estima que en el mundo más de 2000 millones de individuos, principalmente niños, se encuentran infectados por estos parásitos intestinales (1-3). Representan junto con las esquistosomiasis más del $40 \%$ de la carga de enfermedad atribuida a las enfermedades tropicales, la más alta de este grupo de enfermedades después de la malaria (4). Se las ha asociado con anemia, trastornos del desarrollo y el crecimiento, desnutrición crónica y problemas de aprendizaje $(1,3)$. Adicionalmente y dado que estos parásitos se distribuyen solapadamente junto con otras diversas infecciones en los países y áreas endémicas, (5) recientemente se ha propuesto que podría existir un impacto significativo en la potenciación de la carga de la enfermedad por la generación de co-morbilidades; por ejemplo a través de la sumatoria del riesgo o la intensidad de padecer anemia con especial impacto en mujeres embarazadas y niños de áreas altamente endémicas para más de una enfermedad tropical (6-8).

En la actualidad, las geohelmintiasis son catalogadas como enfermedades desatendidas, es decir patologías que persisten con altas prevalencias y que en este caso cuentan con mecanismos de control conocidos de alto costo-efectividad (tool-ready diseases), pero para las que paradójicamente en las últimas décadas ha disminuido el interés para invertir en su control principalmente por falta de voluntad política para intervenir transversalmente en sus determinantes (9). Sin embargo, recientemente las hipótesis de que los geohelmintos podrían tener impactos adicionales sobre la morbilidad general al modificar la aparición, intensidad o curso clínico de otra enfermedad, como por ejemplo actuando potencialmente como un factor de riesgo independiente para la aparición de malaria, sumado al ya mencionado efecto de la co-morbilidad, ha llevado lentamente a reconsiderar su importancia en salud pública como también a plantear la necesidad y el reto de generar estrategias novedosas de intervención conjunta para complejos mórbidos en paquetes de intervención rápida (10-13).

En Colombia estas infecciones siguen siendo importantes. En un estudio realizado en la población de La Virgen, Colombia, sobre tendencias de la prevalencia de las geohelmintiasis entre 1995 y 2005, se observó un descenso de $39,03 \%$ en niños entre cinco y quince años y de $37,5 \%$ en los menores de cinco años. A pesar de la disminución en las prevalencias de los geohelmintos, éstas se mantuvieron altas: $23,91 \%$ y $27,58 \%$ respectivamente (14).

Para la generación de salud en los países en vía de desarrollo se ha planteado un "triángulo que mueve la montaña" que involucra tres vértices primordiales: voluntad política, participación social y generación de conocimiento relevante (15). Con el propósito de contribuir con estos componentes especialmente con una mayor participación social, se recomienda a los agentes de salud comprender el comportamiento humano relacionado con las enfermedades parasitarias, desde la perspectiva de la gente misma (o visión émica) por su potencial importancia en la planificación de los programas de control, los cuales deben ser adecuados al contexto sociocultural, económico y político de las poblaciones para obtener no sólo una mayor aceptación, sino también más probabilidades de éxito. Por lo anterior, es necesario estudiar pero sobre todo reconocer los conocimientos, las actitudes y las prácticas de niños y padres, como un paso inicial para el diseño de programas de educación en salud que les induzca un aprendizaje significativo, para lograr mejores prácticas frente a la infección y una posición más activa, que promueva integralmente una organización social en búsqueda del mejoramiento de las condiciones sanitarias y por ende, de la situación de las infecciones intestinales (16).

\section{Materiales y métodos}

El trabajo se realizó en La Virgen, zona rural del departamento de Cundinamarca, Colombia, distante $100 \mathrm{~km}$ al suroccidente de Bogotá, a $1443 \mathrm{msnm}$ de altitud y con una temperatura promedio de $22^{\circ} \mathrm{C}$. La economía del área se basa en la agricultura de caña de azúcar, cítricos y café. La población total en el momento de la investigación era aproximadamente de 2000 habitantes, 428 en el casco urbano y 1572 en las once veredas aledañas.

\section{Diseño del estudio}

Investigación cualitativa, etnográfica, que permite identificar y contrastar las diferencias entre la perspectiva cultural de la gente (visión émica) y la de los profesionales del sector salud (visión ética). Las herramientas etnográficas facultan "...entender o explorar 
la perspectiva social de los actores, sus definiciones y experiencias, y emplea las expresiones naturalistas del informador como puntos de partida nuevos para descubrimientos y preguntas adicionales" (17), lo cual permite reconstruir las percepciones, las lógicas internas de los saberes y comprender las actitudes y comportamientos derivados.

Los instrumentos que se utilizaron fueron: 1- Observación participante que consistió en identificar las condiciones de saneamiento básico y contrastar lo que decían los entrevistados con sus prácticas cotidianas. 2-Encuesta que permitió un acercamiento inicial a los conocimientos, actitudes y prácticas relacionadas con las geohelmintiasis. 3- Entrevistas semiestructuradas que se emplearon para recoger información a profundidad de los saberes y las percepciones sobre las geohelmintiasis y obtener información de parte de los agentes formales de salud respecto a su percepción sobre los conocimientos de la población. El registro de la información fue escrito, previo consentimiento de los participantes o sus responsables. Para las encuestas y las entrevistas se utilizó el muestreo por conveniencia. El formulario de la encuesta y el de la entrevista se probaron previamente en una zona rural de características similares a las de La Virgen. Se hicieron ocho entrevistas individuales a líderes comunitarios, agentes formales e informales de salud y profesores, diez entrevistas grupales a escolares y padres. 4- Grupos de discusión que propiciaron la interacción de saberes acerca de la dinámica de las geohelmintiasis. Se realizaron por separado con maestros, padres (o cuidadores) y escolares. Se hicieron tres grupos de discusión entre padres, profesores y estudiantes del colegio. 5- Círculos de reflexividad, se hicieron dos con los padres; en este caso los participantes se dividen en dos círculos, el círculo A y el círculo B; en primera instancia el círculo A tiene la palabra y el círculo B escucha, luego se invierten los roles, y los del círculo B complementan o critican las posturas de los del círculo A. 6- Diarios de campo, para tomar notas sobre lo sucedido en cada día de trabajo, las impresiones y sentimientos de cada investigador y cualquier dato que sirviera para contextualizar e interpretar el resto de la información.

Cuando todos los tipos de informantes clave fueron incluidos y la información proveniente de las diferentes fuentes se hizo repetitiva se consideró saturada la muestra total y se dio por finalizado el proceso de recolección. La validez de la información estuvo dada por la triangulación, en el sentido en el que fue entendida por Kemmis como "un control cruzado entre diferentes fuentes de datos: personas, instrumentos, documentos o la combinación de éstos" (18); la confiabilidad por la concordancia interpretativa mediante la participación de varios investigadores.

\section{Consideraciones éticas}

Fue una investigación con riesgo mínimo. Se siguieron las normas para la investigación de la resolución No. 008430 de 1993 del Ministerio de Salud de Colombia, de la Convención Internacional de los Derechos del Niño de 1989 y del Código del menor de 1991. Para las entrevistas se obtuvo el consentimiento informado del escolar cuando era mayor de doce años, o de su representante legal cuando era menor. Se contó con la aprobación de los líderes de la comunidad, del personal del puesto de salud y de las autoridades escolares de la Inspección de La Virgen. La información se codificó para salvaguardar la confidencialidad. El proyecto fue aprobado por el Comité de Ética de la Facultad de Medicina de la Universidad Nacional de Colombia, sede Bogotá.

\section{Procesamiento y análisis de los datos}

El procesamiento de los datos demográficos se hizo con el programa Epilnfo VI y el de los datos cualitativos con el programa Atlas Ti 5.0.66.

\section{Resultados}

De los 130 adultos encuestados el $90,8 \%$ eran mujeres; la edad más frecuente estaba en el rango entre 31-40 años $(40,8 \%)$, seguido por el rango entre $18-30$ años $(26,2 \%)$, el de $41-50$ años $(20,8 \%)$ y en menor proporción los mayores de 51 años (12,3\%). El 68,4\% tenían más de diez años de residencia en la región y el $4,96 \%$ menos de un año. Más de la mitad de los individuos encuestados, 67,9\%, vivían en alguna de las veredas y $32,09 \%$ en el casco urbano. La escolaridad del $57,7 \%$ fue la primaria incompleta, 2,3\% tenían bachillerato y $0,8 \%$ grado técnico. $92,3 \%$ de los niños estaban inscritos en el sistema de salud: $79,8 \%$ en el régimen contributivo y $20,8 \%$ en el subsidiado. La ocupación del $67 \%$ era como jornalero. Al indagar por hacinamiento se encontró que había un promedio de 1,66 individuos por cama en la población estudiada. Los datos correspondientes a las características de la vivienda se resumen en la tabla 1. 
TABLA 1. Características de las viviendas de la población encuestada.

\begin{tabular}{lc}
\hline \multicolumn{1}{c}{ Característica } & Porcentaje $(\mathbf{n}=\mathbf{1 3 0})$ \\
\hline Piso de tierra & 76,9 \\
$\begin{array}{l}\text { Piso de cemento, baldosa, } \\
\text { tabla, otros }\end{array}$ & 23,1 \\
$\begin{array}{l}\text { Ausencia de alcantarillado } \\
\text { Ausencia de recolección de } \\
\text { basuras }\end{array}$ & 91,5 \\
$\begin{array}{l}\text { Acueducto veredal con agua } \\
\text { no potable }\end{array}$ & 87 \\
\hline
\end{tabular}

Tanto en las encuestas como en las entrevistas se abordaron varios aspectos sobre las geohelmintiasis; con las primeras se pretendía tener una línea de base de los conocimientos, actitudes y prácticas de la población sobre estos parásitos y con las segundas, conocer y entender la percepción de la gente sobre estas infecciones. De las entrevistas y los grupos de discusión surgieron las siguientes categorías, que aunque resultan de lo dicho por los entrevistados, se plantean esencialmente en términos éticos (o de los investigadores), para poder correlacionarlos con las categorías preestablecidas de la encuesta: 1- Percepciones sobre los geohelmintos, 2- Nociones sobre el ciclo de vida: en esta emergieron las subcategorías: a) ¿cómo entran al organismo? b) ¿en qué órganos viven? c) ¿dónde se reproducen? d) ¿cómo salen? y e) ¿qué pasa después de que salen del cuerpo? 3- En relación con el punto anterior, la tercera categoría son los riesgos percibidos para adquirir la infección, 4- Población percibida en riesgo de infectarse, 5 - Signos y síntomas desde la perspectiva émica. En este aspecto emergió una subcategoría importante, el "rebote de lombrices", 6.-Terapéutica popular, 7- Medidas preventivas y 8 - Instalaciones sanitarias.

\section{Percepciones sobre los geohelmintos}

En las encuestas, las respuestas a esta pregunta se encuentran resumidas en la tabla 2. En las entrevistas con los niños se evidenció que ellos conocen al Ascaris lumbricoides, al que denominan "la lombriz". Sin embargo, cuando se indaga por la categoría, "los gusanos", es evidente la relación con otros organismos, especialmente, con los "gusanos" de la guayaba que son larvas de insectos. Los padres identifican las lombrices y los gusanos como parásitos intestinales. Los niños y los padres conocen las diferencias entre la lombriz de tierra y el A. lumbricoides. Uno de ellos apunta lo siguiente: "[...] las lombrices de la tierra son más gruesas y las del estómago son más delgaditas y blancas, son como blanquitas".

TABLA 2. Respuestas a la pregunta: “¿Qué son los parásitos?”

\begin{tabular}{lc}
\hline $\begin{array}{c}\text { Característica de la } \\
\text { respuesta }\end{array}$ & Porcentaje* $^{*}(\mathbf{n}=\mathbf{1 3 0})$ \\
\hline Gusanos & 45,7 \\
Lombrices & 44,4 \\
Amebas & 21,5 \\
Animalitos & 8,4 \\
Microbios & 3,2 \\
Bacterias & 1,3 \\
\hline
\end{tabular}

* En algunos casos, la persona respondió más de una opción.

En la categoría de "gusanos" también se incluyen las larvas de Lepidópteros y Coleópteros conocidas en la región con el nombre de "chizas", que se encuentran en los cultivos y en el suelo: "[los gusanos] se encuentran en el estómago y en la tierra y en los palos" "hay uno que se llama gusano pollo.... Ése mantiene en el cafetal y cuando uno va andando ése lo raspa a uno, y lo brota, y lo hincha a uno" "[las diferencias están] en que el gusano pringa, el parásito vive en el estómago y las lombrices viven en la tierra". Los niños y los padres identifican a las tenias o solitarias como parásitos y las describen como "parásitos largos y blancos".

\section{Ciclo de vida}

En esta categoría surgieron cinco subcategorías. 1- ¿Cómo entran al organismo? En las entrevistas y los círculos de reflexividad se observó que los niños saben que las lombrices y los parásitos entran por la boca al ingerir alimentos o aguas contaminadas, o por la piel, por caminar descalzos. Sin embargo, algunos consideran que los geohelmintos pueden entrar también por los orificios naturales del cuerpo como los oídos, la nariz etc., y que los "gusanos" entran por las manos o los pies, pero luego llegan al intestino. 2- ¿En qué órganos del cuerpo viven? La mayoría afirmó que en el estómago o en el intestino, pero otros creen que cuando hay infecciones severas se pueden diseminar por todo el organismo incluyendo riñones y cerebro. 3- ¿Dónde se reproducen? Hay un desconocimiento generalizado sobre 
el ciclo de vida de los parásitos. Según los niños, los geohelmintos se reproducen en el humano hasta alcanzar densidades muy altas en el organismo y los padres creen que los geohelmintos se reproducen en el estómago: “...las lombrices están dentro del estómago de uno y ellas producen huevitos....y eso revienta los gusanos...”. 4. ¿Por dónde salen? Los niños tienen la experiencia de haber visto salir a los geohelmintos en las heces o por otra parte del cuerpo en el caso del A. lumbricoides, algunos mencionan haber visto los gusanos en la materia fecal. 5. ¿Qué pasa después de que salen del cuerpo? Los niños y los padres consideran que los geohelmintos no son viables fuera del organismo humano. Algunos afirman que las "lombrices" que ellos eliminan quedan en el medio ambiente como alimento para animales o como abono para plantas. Algunas expresiones: "....Las gallinas se las comen", ".....Esas se van a abonar la tierra.....". Se identificó además una subcategoría, en la cual reconocen el papel de vectores mecánicos que cumplen las moscas de las "lombrices y los parásitos".

\section{Riesgos percibidos}

En las encuestas los riesgos percibidos fueron similares a los hallados en las entrevistas, estos se observan en la tabla 3. Los entrevistados saben que los factores de riesgo son no lavarse las manos antes de comer y tomar agua sin hervir, consideran que comer guayaba o "frutas pichas" puede ser la causa de la infección por geohelmintos, también es muy común la percepción que comer dulces y leche produce lombrices.: "... los dulces los atraen, la leche también", "esta parte es bastante...contaminada de lombrices... por el sistema de los trapiches... se reproducen mucho tal vez por el dulce".

Todos los informantes creen que la calidad del agua que se consume y la "mala higiene", de los niños y de las viviendas o escuelas, específicamente los baños, y no "ir al médico" periódicamente para la desparasitación son factores de riesgo para adquirir la infección. Las madres también percibieron como un riesgo "la suciedad" y el caminar descalzos. Pero jóvenes de la región señalaron: "Los veía uno [a los niños] andando descalzos y uno les preguntaba que si siempre utilizaban zapatos y nos decían que sí, pero uno los veía andando descalzos."
TABLA 3. Respuestas a la pregunta: "¿Cómo llegan los gusanos o lombrices al cuerpo de los niños?"

\begin{tabular}{lc}
\hline \multicolumn{1}{c}{$\begin{array}{c}\text { Característica } \\
\text { de la respuesta }\end{array}$} & Porcentaje* $^{*}(\mathbf{n}=\mathbf{1 3 0})$ \\
\hline Alimentos sin lavar & 34 \\
$\begin{array}{l}\text { Agua cruda sin hervir o no } \\
\text { tratada }\end{array}$ & 28,7 \\
No lavarse las manos & 28,7 \\
Por estar descalzos & 26,1 \\
No sabe/No responde & 11,1 \\
Por comer dulces & 9,8 \\
Por las frutas y alimentos & 5,9 \\
Por la mala higiene & 2,6 \\
Por el gusano de la guayaba & 1,9 \\
\hline
\end{tabular}

* En algunos casos, la persona respondió más de una opción.

\section{Población percibida como más vulnerable}

Tanto los adultos como los niños reconocen a estos últimos como la población más vulnerable, sin diferencia entre géneros. Los niños creen que se debe a que no se lavan las manos, comen muchos dulces y por "ser más débiles". La auxiliar de enfermería que estuvo a cargo del puesto de salud, relata que por migración de los Ascaris lumbricoides murieron en años pasados varios menores de cinco años.

"En los niños está lo que comúnmente llaman rebote de lombrices. Igual aquí al Puesto de Salud han venido muchos niños con ese problema, que incluso han muerto porque tienen el..., o sea se les salen esos animales por la boca o por cualquier orificio. Bueno, esos casos ya han pasado acá, otra cosa [han muerto] unos tres, más o menos, pero, pues en un término de hace varios tiempos (...). Todo el mundo aquí lo sabe."

Uno de los agentes formales de salud, reconoce que los jóvenes también pueden tener infección por geohelmintos, pero que la mayoría no consultan.

\section{Signos y síntomas}

Cuando se indagó en las encuestas por este aspecto, las respuestas a la pregunta "¿Por qué son dañinos esos gusanos o lombrices para los niños?", se resalta la acción expoliatriz como primer efecto sobre la salud de los niños, los resultados se muestran en la tabla 4. 
TABLA 4. Respuestas a la pregunta: "¿Por qué son dañinos esos gusanos o lombrices para los niños?”

\begin{tabular}{lc}
\hline \multicolumn{1}{c}{$\begin{array}{c}\text { Característica } \\
\text { de la respuesta }\end{array}$} & Porcentaje* (n = 130) \\
\hline $\begin{array}{l}\text { Porque se comen lo que los } \\
\text { niños comen }\end{array}$ & 34,0 \\
Los enferman & 13,7 \\
Retrasan el crecimiento & 9,8 \\
No rinden en el estudio & 7,1 \\
Porque los pone pálidos & 6,5 \\
Les da pereza y sueño & 5,9 \\
Les quita el apetito & 3,9 \\
Dañan el organismo & 2,6 \\
Pueden causar la muerte & 2,6 \\
Dañan los intestinos & 1,9 \\
\hline
\end{tabular}

* En algunos casos, la persona respondió más de una opción.

Los individuos identifican, en general, a los geohelmintos a partir de ciertos signos y síntomas que ellos mismos, o alguien cercano, han padecido. Reconocen como manifestaciones dolor abdominal, náuseas, vómitos, anemia que se refleja en la palidez, diarrea, además de la migración de los parásitos adultos. "Y uno siente que...que algo se le mueve y le revuelve el estómago", "le duele la barriga... y soltura", "Y le dan unas ojeras a uno aquí abajo de los ojos y eso mantiene uno así sin ganas de caminar, sin hacer nada... como todo pálido... y mantiene con una pereza uno". En esta categoría surgió la subcategoría "rebote de lombrices", término que lo usan para $A$. lumbricoides y para amibas. Las personas califican como "rebote" cuando se presentan los signos o síntomas de la infección por estos parásitos.

En el caso de Ascaris lumbricoides lo asocian a la migración de este. "(...) es que por lo menos el niño está durmiendo y ellos empiezan a moverse la nariz, o se sienten ahogados, entonces ahí es cuando la lombriz está andando por el cuerpo y le va saliendo por la nariz. Porque a mi me pasó con un niño mío asî", "... Se van como uniendo y le rebotan a uno el estómago y de ahí le comienza a doler a uno y le da soltura... y ganas de vomitar”, “...ellos comienzan con las muelitas, a mover las muelitas, a babear y los ojitos son diferentes, cambian el color de los ojitos. Los ojitos se colocan como vidriosos, como llorosos". Los niños describieron el rebote de lombrices, como la situación en que "las lombrices están bien crecidas...van subiendo $[y]$ por narices, ojos y orejas salen". Además, “...lo puede matar a uno..., no lo deja respirar...", "Porque uno puede estar por ahí dormido y le comienzan a salir por la nariz".

Pasando a otro tipo de gusanos, los que se encuentran en el cafetal son percibidos por los niños como "venenosos" y que producen picazón y ardor. También hablan de la patología de los geohelmintos: "Cuando uno come los alimentos... uno no se los come... no le alimenta a uno... sino a ellos... los gusanos...los parásitos", "En los intestinos, se alimentan de leche porque es mas nutritiva”, "... [los gusanos del tomate] se van alimentando con la comida que uno va comiendo". Aunque por lo general, los padres identifican los mismos signos y síntomas que los niños, resaltan que la migración puede inducir la muerte. Describen la diarrea con moco o con espuma y reconocen el aumento del volumen abdominal, dormir con los ojos abiertos y "chirriar los dientes" (bruxismo). La auxiliar de enfermería, por su parte, describe la desnutrición como una causa del parasitismo. Y la médica rural informa que los pacientes refieren haber visto los geohelmintos en las deposiciones y se quejan de prurito anal.

\section{Acciones terapeúticas}

Las respuestas en la encuesta a la pregunta, “¿Qué prefiere darle a sus hijos (y/o a los niños que cuida) contra los gusanos/lombrices?, se muestran en la tabla 5 . Sobresale el hecho que más de la mitad de los encuestados utiliza los medicamentos que les ordena el médico; sin embargo, siguen utilizando remedios naturales. Los niños y las madres manifiestan que cuando se produce sintomatología los llevan al médico para que les manden un "purgante" e incluso reconocen los nombres de los medicamentos: "El Abendazol y la Biperacina los mata y los saca". Para "el rebote de lombrices" el tratamiento es de carácter tradicional. Las madres para "aplacarlos" administran paico (Chenopodium ambrosioides), tomado en ayunas por una semana, en infusión de ramas, raíces u hojas al que algunas veces le agregan limón. Algunos de ellos manifiestan que con esta medicina natural se eliminan las "lombrices"y los "gusanos". "Paico. Ése se echa en agua, se hierve el agua y después se la toma uno, es como la hierbabuena, sin comer nada", "Para juntas cosas [gusanos y lombrices] sirve el paico". 
TABLA 5. Respuesta a la pregunta: "¿Qué prefiere darle a sus hijos (o a los niños que cuida) contra los gusanos/lombrices"?

\begin{tabular}{lc}
\hline \multicolumn{1}{c}{$\begin{array}{c}\text { Característica } \\
\text { de la respuesta }\end{array}$} & Porcentaje* $^{*}(\mathbf{n}=\mathbf{1 3 0})$ \\
\hline Purgantes (sin especificar cual) & 38 \\
Lo que el médico formule & 30,7 \\
Albendazol & $7,1 \%$ \\
Paico & 8,4 \\
No le da nada & 15,8 \\
\hline
\end{tabular}

* En algunos casos, la persona respondió más de una opción.

Otra medida terapéutica es tomar ajo machacado con leche durante una semana, o poner collares de ajo a los niños, especialmente para evitar que se produzca el "rebote de lombrices". En este caso, para "aplacar" los gusanos, mientras se llevan los niños al médico, se tienen otros remedios caseros: "una cuñada me dijo ¿tiene vinagre?(...)Yo le dije: si, yo tengo. Me dijo: úntele en los piecitos y de la cabecita a los pies y en la cabecita, y únjale vinagre en el cuerpo y yo le hice eso, y la yerbabuena. Le hice agüita de yerbabuena y lo puse a dormir. Y le aplacaron dentro de lo que dijo ella. Yo digo que el paico y eso no mata las lombrices, las aplaca, las aplaca mientras uno lo lleva al médico".

Las madres coinciden con sus hijos en cuanto al conocimiento sobre la terapéutica, aunque informan que prefieren llevarlos al médico y que les sean formuladas medicinas alopáticas, en detrimento de las terapéuticas tradicionales. Pocas madres (tres) mencionaron haber tenido experiencias negativas con los remedios naturales. Para la auxiliar de enfermería y la promotora de salud es frecuente el hecho que las madres den a sus hijos infusiones de paico al menos dos veces al año. En cambio la médica rural dijo no conocer ese tipo de tratamientos populares. Las tres coinciden en que las madres llevan a sus hijos dos veces al año al médico para ser desparasitados.

\section{Medidas preventivas}

Los niños y adultos saben cuáles son los hábitos perjudiciales, que idealmente no deberían tener para así prevenir las geohelmintiasis, por ejemplo, no lavarse las manos, tomar agua cruda y no lavar los alimentos; sin embargo, la no asociación de la tierra y los geohelmintos, hace que para ellos esa medida no tenga significado. Además hay situaciones socioeconómicas y de valores que priman: el baño de la escuela está dañado y se ven forzados a ir al monte; en las viviendas no hay tazas sanitarias o no tienen pozo séptico, "el alcalde no me ha ayudado" y "somos pobres". Varios adultos suelen percibir "la purga" (ya sea con remedios caseros o alopáticos) más como una práctica preventiva que curativa. Por otra parte, es relevante para la prevención la reflexión que los niños hacen sobre lo que sucede con los parásitos cuando salen del cuerpo: "[mueren porque] no están acostumbrados al aire puro" y "porque ya no tienen alimentación". Pero no tienen en cuenta que los huevos sobreviven en el suelo y su paso por éste es fundamental para que se vuelvan infectantes para el humano.

\section{Instalaciones sanitarias}

Cuando se les preguntaba en la encuesta sobre las instalaciones sanitarias, el 60,8\% de los cuidadores dicen que los niños van al monte cuando se encuentran en la casa y que cuando estaban en la escuela $81,5 \%$ las usaban, pero los investigadores observaron que en la mayoría de las escuelas las instalaciones eran inadecuadas, por tanto los niños iban al monte. Cuando se entrevistaron profesores y líderes comunitartios, más la comparación de lo dicho versus lo observado, se estableció que la mitad de las casas tienen tazas sanitarias o similares, pero no de buena calidad, por tanto la mayoría de la población dispone las excretas a campo abierto.

\section{Discusión y conclusiones}

Tanto las respuestas a la encuesta como las percepciones expresadas por los participantes en las entrevistas y los círculos de reflexividad tienen una alta concordancia. Es claro que las categorías parásitos, gusanos y lombrices no coinciden estrictamente con su acepción en el conocimiento biomédico, pues corresponden a construcciones socioculturales distintas, las cuales sólo tienen un sentido comprensible para los miembros de la cultura correspondiente (19-20). Si se tiene en cuenta que tales nociones se manifiestan en percepciones, actitudes y comportamientos específicos frente al cuidado de la salud y al tratamiento de la enfermedad, reconocerlas y entender su significado es importante en el momento de transformar prácticas (21-22). Por esto, en virtud de la idea que tienen de "gusano", que surge posiblemente de las similitudes en tamaño y forma que tienen los gusanos de la guayaba y los adultos de Uncinaria y Trichuris trichiura, los niños consideran 
que una forma de evitar la infección es abstenerse de consumir alimentos como la guayaba y el tomate, hecho que resulta contraproducente en tanto elimina o limita una fuente de alimento producida en la región. Desafortunadamente, esta percepción es compartida por los cuidadores. Además, los niños incluyen dentro de esta categoría larvas de insectos que les producen reacciones alérgicas o urticantes cuando las tocan. Los padres consideran que los gusanos son una fase del desarrollo de A. lumbricoides, que además estos parásitos ponen los huevos en el intestino, luego se convierten en gusanos y finalmente en lombrices, las cuales una vez salen del organismo mueren y no constituyen un riesgo; los padres y los niños incluso sostienen que las lombrices sirven de abono para las plantas o de alimento para las gallinas. Estas creencias sumadas a la falta de instalaciones sanitarias adecuadas, explicarían por qué en esta región aún existen altas prevalencias de parásitos intestinales incluidos los geohelmintos; solo los profesores consideran esas creencias como un factor de riesgo, diferente a lo descrito por Mello y col. (23) y similar a lo encontrado por Rousham (24).

En este estudio se evidenció que el factor de riesgo percibido como más importante es el consumo de alimentos sin lavar, agua sin hervir, seguido por no lavarse las manos, caminar descalzos, basuras, consumo de alimentos dulces y leche e incluso la producción de panela y el consumo de algunas frutas. En Bangladesh y Nepal se describieron estas mismas percepciones, especialmente de los últimos riesgos descritos. Llama la atención que a pesar de ser culturas tan distantes compartan estas mismas creencias. $(24,25)$. El reconocimiento de las nociones de la gente de las comunidades permite identificar la causa de ciertas prácticas de riesgo e identificar falencias en información. Si se les brinda información clara y significativa del ciclo de vida de los parásitos a través del diálogo de saberes distintos, la transformación de las prácticas tendrá sentido para ellos y por tanto será mas exitosa. Asimismo, hay que comprender qué es posible realizar en la región, de acuerdo a sus recursos socioeconómicos, culturales, naturales y legales. También es necesario reconocer al otro y con él plantear alternativas de solución a los diversos problemas de salud para que haya una identificación y apropiación tanto de los fines que se buscan como de los medios a emplear.

En La Virgen se dice que las vías de entrada de los parásitos son la oral, por el agua y los alimentos conta- minados, y por los pies, creencias que están de acuerdo al conocimiento biológico de los parásitos y es similar a lo que creen en comunidades rurales del Brasil (26).

En cuanto a las manifestaciones clínicas, en el presente estudio se subraya el "rebote". Los informantes hablan de "rebote de lombrices" o "rebote de amibas" cuando es producida por A. lumbricoides o por Entamoeba histolytica, respectivamente, para denotar cuando se están produciendo manifestaciones clínicas de la presencia de los parásitos. Las personas también lo asocian con la salida de los gusanos adultos por orificios naturales o con la materia fecal, Muchos niños y adultos tienen la experiencia de haber expulsado "gusanos" con las heces. Los padres informan la experiencia de haber visto en las deposiciones $A$. lumbricoides y otros "gusanos". El término "rebote" se ha encontrado que es utilizado casi solamente en Colombia. Algunas de las manifestaciones percibidas por la población como la desnutrición, aumento del volumen abdominal y bruxismo, han sido encontradas también por otros autores $(24,26)$. Si bien la gente de la comunidad reconoce el parásito asociado a la enfermedad, las explicaciones respecto a la causa del padecimiento no corresponden directamente con la etiología expuesta desde la biomedicina.

Las manifestaciones de la enfermedad relatadas por la gente no sólo hablan de signos y síntomas en un cuerpo en términos biológicos, también describen las dolencias de un cuerpo que se piensa y se construye dentro de unos imaginarios sociales (27). Los programas de control podrán identificar los aspectos en los que deben centrarse y la manera de hacerlo, al reconocer las preocupaciones particulares de la comunidad sobre su bienestar, es decir su percepción de salud.

Con respecto al tratamiento, es ampliamente generalizado que las madres les procuren a los niños paico, entre otros antes de llevarlos al médico. Algunos informantes de la población de La Virgen relatan que tanto el paico como el albendazol inducen la salida de los gusanos en la materia fecal. López de Guimaraes y col. (28) encontraron que la eficacia terapéutica del zumo de paico y del albendazol fue similar, con la ventaja que el paico fue $100 \%$ eficaz para Hymenolepis nana. El uso de vinagre como parasiticida, se registró también en Brasil (29). En La Virgen, las prácticas de medicina tradicional tienen un fuerte arraigo, lo cual les provee de legitimidad; además son fáciles de adquirir porque 
se encuentran en la zona y se pueden combinar con las terapéuticas de la medicina occidental.

El hecho que las madres reconozcan el papel del médico y lleven a los niños cada seis meses para desparasitarlos posiblemente ha sido la causa de la disminución de la morbilidad severa y la prevalencia, así lo reconoce uno de los agentes formales de salud cuando menciona que hace unos años hubo muertes por la migración de los A. lumbricoies y que ahora ya no se presentan.

Las geohelmintiasis no se pueden entender exclusivamente desde el aspecto biológico; es necesario mirar el contexto socio-cultural que genera una estructura epidemiológica, lo que implica determinantes específicos de la enfermedad (30). Los programas de educación en promoción y prevención siempre encontrarán un conjunto de saberes previos que deberían ser entendidos por quienes trabajan en ellos para que no sólo sean culturalmente apropiados sino para que respondan a la realidad en que viven los grupos sociales. Las sociedades construyen una idea del problema y una solución, que no se corresponde estrictamente con las definiciones de la biomedicina, sino que son construcciones sociales que involucran sentimientos y actitudes con respecto a la enfermedad, (19-20), como la asociación de la presencia de "los gusanos" con el dulce.

En otras palabras, la convivencia con una patología lleva a que la comunidad desarrolle conocimientos, comportamientos y sentimientos específicos frente a la enfermedad y tratamientos que se articulan a sus sistemas terapéuticos tradicionales (31). Es decir, son un conjunto de saberes que conforman estilos de vida comunitarios y que hacen parte de una lógica de mantenimiento biológico, económico y social de la comunidad (32). Antes de cualquier evaluación epidemiológica, la población de La Virgen ya había hecho una valoración del riesgo y desarrollado un modo de control por sí misma aún cuándo no sea de alta eficacia.

Construir programas de educación sin tener en cuenta la participación de las comunidades locales hace que sean poco efectivos en términos de la aceptación; es necesario empezar por reconocer los términos y conceptos asociados a las infecciones y el significado que tienen para esa sociedad. Hay maneras de clasificar la naturaleza, organizarla en conceptos y adscribirles significados en cada comunidad (32). La comprensión de tales términos por parte de quienes apliquen los programas permitirá un diálogo de saberes que hará posible una interacción más fluida con la comunidad. Es importante entonces tener en cuenta los saberes populares y no pretender reemplazar el conocimiento cotidiano en tanto éstos tienen características y aplicabilidades distintas (20,31). No obstante, la exclusión e inclusión de ciertas ideas en el conocimiento cotidiano a partir de la orientación del conocimiento científico, puede permitir la transformación de muchas prácticas de riesgo.

Este estudio muestra que los factores de riesgo no deben ser exclusivamente considerados en términos de individuos o de determinantes sociales desarticulados (sexo, edad, nivel socioeconómico), sino dentro de un contexto sociocultural con un potencial explicativo fundamental que permite entender las condiciones bioculturales en que se instalan y prosperan estas infecciones (20), más allá de ser sólo un indicador de riesgo. Por ello, identificar y entender los conocimientos, actitudes y prácticas de la gente permite acercarse a la lógica popular sobre los fenómenos de saludenfermedad, comprender por qué la gente actúa de una manera particular y así proponer posibles cursos de acción para la prevención de la enfermedad y la promoción de la salud.

\section{Agradecimientos}

A la comunidad rural de La Virgen, en especial a Tobías Castiblanco, al Dr. Germán González y a los estudiantes de medicina Paula Díez, María Amelia Bueno y Carlos Valencia.

\section{Conflicto de intereses y fuente de financiación}

Los autores declaran no tener conflicto de intereses. Investigación financiada por la División de Investigaciones de la Universidad Nacional de Colombia. Sede Bogotá (DIB).

\section{Referencias}

1. World Health Organization. Prevention and control of Schistosomiasis and Soil Transmitted Helminth Infections. Preliminary estimates of the number of children treated with albendazole or mebendazole. Weekly Epidemiological Record. 2006; 81:145-64.

2. Cromptom D W. How Much Human Helminthiasis is There in the World? The Journal of Parasitology. 1999;85:397-403.

3. De Silva N R, Brooker S, Hotez P J, Montresor A, Engels D, Savioli L. Soil-Transmitted Helminth Infections: Updating the Global Picture. Trends In Parasitology. 2003;19:547-551.

4. Chan MS. The Global Burden of Intestinal Nematode InfectionsFifty Years On. Parasitology Today. 1997;13:438-443. 
5. Petney T N, Andrews R H. Multiparasite Communities in Animals and Humans: Frequency, Structure and Pathogenic Significance. International Journal of Parasitology. 1998;28:377-393.

6. Brooker S, Clements A, Hotez P, Hay S, Tatem A, Bundy D. The Co-Distribution of Plasmodium and Hookworm among African School Children. Malaria Journal. 2006;5:99.

7. Fenn B, Morris S S, Black R E. Comorbidity in Childhood in Northern Ghana: Magnitude, Associated Factors, and Impact on Mortality. International Journal of Epidemiology. 2005;34:368-375.

8. Hilliguier S D, Booth M, Muhangi L, Nkurunziza P, Kihembo M, Kakande M. Malaria and Helminth Co-infection in a Semi-urban Population of Pregnant Women in Uganda. Journal of Infectious Disease. 2008;198:920-927.

9. Ehrenberg J P, Ault S K. Neglected Diseases of Neglected Populations: Thinking to reshape the Determinants of Health in Latin America and the Caribbean. BMC Public Health. 2005;5:e119.

10. Nacher M. Worms and Malaria: Noisy Nuisances and Silent Benefits. Parasite Immunology. 2002;24:391-93.

11. Helmby H. Helminths and our Immune System: Friend or Foe? Parasitology International. 2009;58(2):121-27.

12. Fernández JA, Idrovo AJ, Cucunubá ZM, Reyes P. Validez de los Estudios de Asociación entre Geohelmintos e Incidencia de Malaria: ¿Deberían Impactar las Políticas de Salud? Revista Brasileira de Epidemiologia. 2008;11:365-78.

13. Hotez P J, Molyneux DH, Fenwick A, Ottesen E, Ehrlich Sachs S, Sachs J D. Incorporating a Rapid-impact Package for Neglected Tropical Diseases with Programs for HIV/AIDS, Tuberculosis, Malaria. PLoS Medicine. 2006;3:e102.

14. Fernández-Niño J A, Reyes-Harker P, Moncada-Álvarez L I, López M C, Chaves M P, Knudson A. Tendencia y Prevalencia de las Geohelmintiasis en La Virgen, Colombia 1995-2005. Revista de Salud Pública. 2007;9:289-296.

15. Wasi P. "Triangle that Moves the Mountain" and Health Systems Reform Movement in Thailand. Human Resources for Health Development Journal. 2000;4:106-110.

16. Vera L, Amador L R. Las Bases del Aprendizaje Eficiente: Comprender más que Saber. pp.73-82. In: Rojas E, Vera L, Madiedo N. Reflexiones sobre educación universitaria III. Bogotá. Unibiblos. Universidad Nacional de Colombia. 2007.

17. Mead, Crawford L, Eckert J K, Zimmermal S, Schumacher J G. Sociocultural Aspects of Transitions from Assisted Living for Residents with Dementia. The Gerontologist. 2005;45:115-123.

18. Kemmis S. Mejorando la Educación Mediante la Investigaciónacción. In: Salazar M C (comp). La Investigación- Acción Participativa: Inicios y Desarrollos. Edición Popular. Madrid. 1992. 135-174.

19. Berlin E A, Berlin B, Lozoya X, Meckes M, Tortoriello J, Villarreal M L. The Scientific Basis Gastrointestinal Herval Medicine among the Highland Maya of Chiapas, Mexico. En: Nader L. Compiladora. Naked Science: Anthropological Inquiry into boundaries, power and knowledge. Nueva York. Routledge. 1996.

20. Costa-Neto E M, Magalhães H F. The Ethnocategory "Insect" in the Conception of the Inhabitants of Tapera County, São Gonçalo dos Campos, Bahia, Brazil. Anais da Academia Brasileira de Ciências. 2007;79:239-249.

21. Anahi S Y. Búsqueda de la Salud en Comunidades Mbya- Guaraní de la Provincia de Misiones, Argentina. Una Aproximación Etnográfica a través del Estudio de Casos. Anthropology and Health Journal. 2008;1:35-43.

22. Anahi S Y. Una Revisión en Torno a los Estudios de Enfermedades Gastrointestinales. En Busca de Nuevas Alternativas para el Análisis de Procesos de Salud-Enfermedad. Salud Colectiva. 2009;5:49-62.

23. Mello D A, Pripas S, Fucci M, Santoro M C, Pedrazzani E S. Helmintoses Intestinalis. Conhecimentos, Atitudes e Percepcoes da Populacao. Revista de Saúde Pública. 1988;22:140-149.

24. Rousham E K. Perceptions and Treatment of Intestinal Worms in Rural Bangladesh Local Differences in Knowledge and Behavior. Social Science and Medicine. 1994;39:1063-68.

25. Williams-Blangero S, Subedi J, Upadhayay R P, Manral D B, Khadka K, Jirel S. Attitudes Towards Helminthic Infection in the Jirel Population of Eastern Nepal. Social Science and Medicine. 1998;47:371-79.

26. Gomes Dos Santos M, Moreira M M, Malaquias M L, Schall V T. Health Education in First Level Schools at the Outskirts of Belo Horizonte, Minas Gerais State, Brasil: II. Awareness, Knowledge and Prevalence of Helminthic Diseases among First Grade Teachers and Pupils. Revista del Instituto de Medicina Tropical de Sao Paulo. 1993;35:573-579.

27. Good B. Medicina, Racionalidad y Experiencia. Una Perspectiva Antropológica. Barcelona. Ediciones Bellaterra. 2003.

28. López de Guimaraes D, Neyra R S, Romero J H. Ascaridiasis: Comparación de la Eficacia Terapéutica entre Paico y Albendazol en Niños de Huaraz. Revista de Gastroenterología. 2001;21.

29. Disponible en: http://sisbib.unmsm.edu.pe/bVrevistas/gastro/ Vol_21N3/ascaridiasis.htm. Consultado el 16 de junio de 2009.

30. Gomes dos Santos M, Guimaraes A, Moreira M. Conhecimiento e Uso da Medicina Alternativa entre Alumos e Profesores de Primero Grau. Revista de Saúde Pública. 1995;29:221-27.

31. Asaolu S O, Ofoezie I E. The Role of Health Education and Sanitation in the Control of Helminth Infections. Acta Tropica. 2003;86:283-94.

32. Simoes C. Epidemiology and Anthropology an Integrated Approach dealing with Bio-Socio-Cultural Aspects as Strategy for the Control of Endemic Diseases. Memórias do Instituto Oswaldo Cruz. 1998;93(1):59-62.

33. Rozenberg B. O Saber Local e os Dilemas Relacionados à Validação e Aplicabilidade do Conhecimento Científico em Áreas Rurais. Cadernos de Saúde Pública. 2007;23(1):97-105. 